Información farmacológica

RETIROS DEL MERCADO Advertencia de no consumir siete productos fitoterapéuticos.

REACCIONES ADVERSAS Inhibidores de la enzima de conversión de la angiotensina, diuréticos y antiinflamatorios no esteroideos: un trío peligroso; reacciones psiquiátricas a los inhibidores de la COX-2; neumopatía intersticial con la nitrofurantoína; hiperglucemia y diabetes con la olanzapina; riesgo de tromboembolia venosa con las combinaciones de ciproterona y etinilestradiol; anticonceptivos orales y riesgo de cáncer cervical en mujeres infectadas por papilomavirus humanos de alto riesgo; la suspensión de la paroxetina puede producir síntomas graves; pancreatitis asociada al alendronato; trastornos prostáticos con loratadina.

USO RACIONAL Utilización de las insulinas en la diabetes de tipo 2; fracasos terapéuticos del antimonio pentavalente; riesgos y beneficios del prazicuantel en el tratamiento de la esquistosomiasis en mujeres lactantes y embarazadas; información de seguridad importante para pacientes tratados con celecoxib; el tamoxifeno en la prevención del cáncer de mama y el riesgo de fenómenos tromboembólicos y cáncer endometrial.

ENMIENDAS A LA ROTULACIÓN/ CAMBIOS DE FORMULACIÓN

DECISIONES DIVERSAS

EVALUACIÓN DE NUEVOS PRODUCTOS

POLÍTICAS DE MEDICAMENTOS
Revisión de las precauciones a tomar con la acarbosa, el zafirlukast y la vincristina; cambios importantes en el prospecto de enoxaparina sódica inyectable; se refuerzan las secciones de "Advertencias" y "Contraindicaciones" de la ziprasidona; se enmienda el prospecto de la clozapina para reflejar su asociación a la miocarditis.

La FDA aprueba la comercialización restringida del alosetrón.

¿Es necesaria otra estatina (rosuvastatina)?; otro análogo de las prostaglandinas (travoprost) para el tratamiento del glaucoma.

Negociaciones entre los países del Caribe y la industria farmacéutica para bajar los precios de los antirretrovíricos en la región; nuevos países participantes en el Programa Internacional de Farmacovigilancia de la OMS.

\section{RETIROS DEL MERCADO}

Advertencia de no consumir siete productos fitoterapéuticos (Canadá)

Las autoridades sanitarias de Canadá (Health Canada/Santé Canada: HC/ SC) advierten a la población de que no debe consumir siete productos fitoterapéuticos fabricados en los Estados Unidos de América (EE. UU.) por BotanicLab, porque contienen, sin declararlo, fármacos que necesitan prescripción médica y que pueden tener repercusiones graves sobre la salud si no se toman bajo supervisión médica. Los siete productos, todos ellos en forma de cápsulas, están comercializa- dos como medicamentos tradicionales chinos para diferentes indicaciones y son vendidos por diferentes distribuidores y profesionales sanitarios, así como por correo, teléfono e Internet. Los productos y los fármacos que contienen son:

- Arthrin ${ }^{\circledR}$ : indometacina, dietilestilbestrol y alprazolam,

- Osporo $^{\circledR}$ : indometacina y dietilestilbestrol,

- Poena ${ }^{\circledR}$ : indometacina,

- Neutralis ${ }^{\circledR}$ : indometacina,

- 5OA Plus ${ }^{\circledR}$ : indometacina y alprazolam,

- RA Spes ${ }^{\circledR}$ : indometacina y alprazolam, y

- Hepastat ${ }^{\circledR}$ : indometacina.
HC/SC aconseja a quienes hayan tomado estos productos que dejen de hacerlo y consulten a su médico.

\section{REACCIONES ADVERSAS}

Inhibidores de la enzima de conversión de la angiotensina, diuréticos y antiinflamatorios no esteroideos: un trío peligroso (Nueva Zelandia)

El Comité Asesor de Reacciones Adversas a los Medicamentos (Adverse Drug Reaction Advisory Committee: ADRAC) de Australia ha aportado pruebas de que esta combinación de fármacos puede precipitar la insuficien- 
cia renal. En 1999 el ADRAC observó que en 46 de 78 notificaciones de insuficiencia renal aguda los pacientes estaban tomando combinaciones de uno o más de los siguientes fármacos: antiinflamatorios no esteroideos (AINE), diuréticos e inhibidores de la enzima de conversión de la angiotensina (IECA); siete de ellos estaban tomando las tres clases de fármacos. La base de datos del ADRAC contiene también 56 casos de insuficiencia renal o empeoramiento de la insuficiencia renal asociados al celecoxib, 24 de los cuales estaban tomando también un diurético y un IECA o un antagonista del receptor de la angiotensina II. Los pacientes con trastornos que predisponen a la insuficiencia renal necesiten a menudo IECA o diuréticos y no deberían tomar simultáneamente AINE o inhibidores de la ciclooxigenasa-2 (COX-2). Los pacientes que estén tomando los tres tipos de fármacos y sufran algún trastorno predisponente, como deshidratación o diarrea, deberían dejar de tomar AINE e inhibidores de la COX-2, y además se debería vigilar cuidadosamente su función renal y las concentraciones plasmáticas de potasio. Las dosis de otras medicaciones deberían ser ajustadas en función de su estado. Los ancianos son particularmente susceptibles a la insuficiencia renal inducida por esta combinación de fármacos. La mayoría de ellos sufre ya algún deterioro de la función renal. Además los ancianos son propensos a la deshidratación e hipotensión inducidas por los diuréticos, y su ingesta de líquidos es a menudo insuficiente.

\section{Reacciones psiquiátricas a los inhibidores de la COX-2 (Nueva Zelandia)}

Durante el primer año de vigilancia (hasta febrero de 2002) de los inhibidores de la COX-2 en el Programa de Vigilancia Intensiva de Medicamentos (Intensive Medicines Monitoring Programme) de Nueva Zelandia, se han recibido 291 notificaciones relacionadas con el celecoxib y 149 con el rofecoxib, trece de ellas ( 11 y 2 , respectivamente) sobre reacciones psiquiátricas agudas: varios casos de confusión, de- presión y alucinaciones, uno de ansiedad, uno de alteraciones del pensamiento y otro de exacerbación de una psicosis maníaco-depresiva. La mayoría de las notificaciones correspondieron a ancianos y los casos observados en mujeres fueron más numerosos que en hombres, aunque hay que tener en cuenta que estos fármacos se prescriben con mayor frecuencia a mujeres. Todas estas reacciones psiquiátricas se resolvieron rápidamente tras la suspensión del tratamiento. También hay en la literatura algunos informes que indican que pueden ocurrir reacciones similares con los AINE convencionales. Los médicos deben estar atentos ante la posibilidad de que se produzcan reacciones psiquiátricas con los inhibidores de la COX-2 o los AINE.

\section{Neumopatía intersticial con la nitrofurantoína (Nueva Zelandia)}

La aparición reciente en Nueva Zelandia de un caso mortal de neumopatía intersticial debido al tratamiento prolongado con nitrofurantoína señala la necesidad de vigilar su posible toxicidad pulmonar. Se sabe que la nitrofurantoína puede causar reacciones pulmonares tanto agudas como crónicas. Los tratamientos prolongados pueden producir neumopatía intersticial y fibrosis pulmonar. Por lo tanto, en pacientes con tratamientos prolongados se debe vigilar la función pulmonar e interrumpir la administración del fármaco ante la aparición de los primeros signos de lesión pulmonar. Los síntomas suelen mejorar rápidamente, pero las alteraciones radiográficas pueden persistir.

\section{Hiperglucemia y diabetes con la olanzapina (Reino Unido)}

La olanzapina, un antipsicótico atípico, puede alterar el metabolismo de la glucosa. En el Reino Unido se han notificado 40 casos de hiperglucemia, diabetes o exacerbación de la diabetes. En cuatro se produjo cetoacidosis o coma y uno fue mortal. Todavía no se ha identificado el mecanismo exacto responsable de estos efectos, pero se sabe que tras el inicio del tratamiento con olanzapina se puede producir un marcado y rápido aumento de peso, que puede preceder la aparición de la hiperglucemia o la exacerbación de una diabetes preexistente. Se ha enmendado la información sobre el producto para incluir la información pertinente sobre estos efectos.

\section{Riesgo de tromboembolia venosa con las combinaciones de ciproterona y etinilestradiol (Nueva Zelandia)}

Las píldoras de estrógenos y ciproterona (Diane 35/35D, Estelle 35/35D) se usan en el tratamiento de trastornos causados por el exceso de andrógenos, como el acné grave, y son anticonceptivas. El Comité de Reacciones Adversas a los Medicamentos (Medicines Adverse Reactions Committee) de Nueva Zelandia ha advertido de que el riesgo de tromboembolia venosa con los anticonceptivos orales (AO) que contienen acetato de ciproterona es al menos tan elevado como con los AO de tercera generación, y recuerda que las píldoras de etinilestradiol y ciproterona solo están indicadas en mujeres, para el tratamiento de enfermedades dependientes de los andrógenos, como el acné grave y el síndrome poliquístico ovárico, así como para proporcionar anticoncepción oral a estas pacientes. Todas las pacientes que estén tomando esta medicación en la actualidad deberían ser revisadas en la próxima consulta para confirmar que el tratamiento está indicado, y se les debería advertir del riesgo de tromboembolia venosa, de sus síntomas y de las situaciones que aumentan el riesgo.

\section{Anticonceptivos orales y riesgo de cáncer cervical en mujeres infectadas por papilomavirus humanos de alto riesgo (Reino Unido)}

El Departamento de Salud del Reino Unido ha enviado a todos los profesionales sanitarios un comunicado urgente en el que señala que un estudio reciente publicado en Lancet (2002) 
aporta datos adicionales que, aunque no son concluyentes, indican que los AO pueden contribuir a la aparición de cáncer cervical en mujeres infectadas por papilomavirus (HPV) de alto riesgo. El estudio informa de una asociación entre el aumento del riesgo de cáncer cervical y el aumento de la duración del consumo de $\mathrm{AO}$ (riesgo tres veces mayor tras 5 a 9 años de consumo, y cuatro veces mayor tras $10 \mathrm{o}$ más años). Con los datos actuales es difícil afirmar si el principal factor precipitante del cáncer cervical es el consumo de AO, la actividad sexual, el tipo de HPV o la duración de la infección. Además, los estudios originales se realizaron en mujeres de países en desarrollo que no disponen de programas adecuados de detección del cáncer cervical. Por consiguiente, el comunicado advierte que todas las mujeres sexualmente activas, y en especial las que están en tratamiento prolongado con $\mathrm{AO}$, deben someterse regularmente a la realización de frotis cervicales. Los beneficios de los AO superan los riesgos en la gran mayoría de las mujeres.

\section{La suspensión de la paroxetina puede producir síntomas graves (EE. UU.)}

La FDA ha publicado una advertencia en la que señala que la suspensión de la paroxetina puede producir síntomas de abstinencia graves, capaces de conducir a la dependencia, entre ellos malos sueños, parestesias y mareos. Si estos síntomas son intolerables, el médico debe considerar la posibilidad de volver a administrar el fármaco. Los pacientes necesitan ser advertidos de que no deben interrumpir bruscamente la toma de antidepresivos, y los médicos deben reducir las dosis gradualmente, observando la posible aparición de síntomas de abstinencia.

\section{Pancreatitis asociada al alendronato (Canadá)}

El Programa Canadiense de Vigilancia de las Reacciones Adversas a los Fármacos (Canadian Adverse Drug Re- action Monitoring Program) ha sido notificado de seis casos de pancreatitis asociados al tratamiento con ácido alendrónico entre diciembre de 1995 (fecha de la comercialización del fármaco) y agosto de 2001. Basándose en estos casos, resulta difícil establecer una relación causal, pero uno de ellos solo estaba recibiendo ácido alendrónico y la complicación se resolvió al suspender la medicación. Además, la paciente no presentaba ningún otro factor de riesgo de pancreatitis. Se destaca la necesidad de seguir notificando otros casos sospechosos de pancreatitis asociada al ácido alendrónico, para que se pueda evaluar mejor esta posible reacción adversa al fármaco.

\section{Trastornos prostáticos con loratadina}

La loratadina es un antihistamínico tricíclico con actividad selectiva frente a los receptores $H_{1}$ periféricos, indicado en el tratamiento de la rinitis alérgica y de otros síntomas de la fiebre del heno. Entre un total de 7937 notificaciones de efectos adversos, ha habido 5 de trastornos prostáticos y 1 de agravación del prostatismo. Tres mejoraron con la suspensión del tratamiento y en uno no hubo recurrencia al volver a administrarlo. Todos estaban tomando loratadina combinada con pseudoefedrina. Recientemente también se han notificado 12 casos de glaucoma que, junto con los casos de prostatismo, indican que la loratadina tiene efectos anticolinérgicos. La frecuencia de la retención urinaria oscila entre el 0,1 y el 0,4\% con los antihistamínicos sedantes y entre el 0,2 y el $0,3 \%$ con los antihistamínicos "no sedantes". Sin embargo, con la loratadina de acción prolongada, la frecuencia de este efecto ha sido del 1,1\%.

\section{USO RACIONAL}

\section{Utilización de las insulinas en la diabetes de tipo 2 (Australia)}

Un estudio realizado en el Reino Unido ha revelado que la diabetes de tipo 2 es una enfermedad progresiva y que los pacientes necesitan dosis crecientes y un número cada vez mayor de hipoglucemiantes orales. Muchos acaban necesitando insulina. Sin embargo, los pacientes suelen ser reacios a inyectarse insulina, y los médicos a prescribirla, por lo que la decisión de iniciar la insulinoterapia se puede aplazar durante años. La concentración deseable de hemoglobina glucosilada es del 7\% y el límite para iniciar acciones está fijado en el $8 \%$, pero muchos pacientes presentan valores más elevados durante muchos años. En general, cuanto mayores sean la glucemia y la concentración de hemoglobina glucosilada, mayores son los beneficios de su reducción. Una vez que se toma la decisión de iniciar la insulinoterapia, tanto los pacientes como los médicos suelen verse sorprendidos por lo fácil que resulta el tratamiento y ambos se sienten mucho mejor (físicamente los pacientes y profesionalmente los médicos). Además, los pacientes suelen agradecer la reducción del número de comprimidos que tienen que tomar y de su costo.

\section{Fracasos terapéuticos del antimonio pentavalente (Brasil)}

La Unidad de Farmacovigilancia ha sido notificada de casos graves de ineficacia terapéutica en pacientes con leishmaniasis tratados con antimoniato de meglumina, cuya causa más probable es la prescripción de dosis subterapéuticas. Esto podría explicarse por el hecho de que dichas prescripciones se estén basando únicamente en la lectura del prospecto del medicamento, que dice que el producto contiene una concentración de 1,5 g/5 mL. Sin embargo, la Fundación Nacional de Salud (FUNASA) de Brasil recomienda que la dosis se calcule con base en la concentración de 405 $\mathrm{mg}$ de antimonio pentavalente por cada $5 \mathrm{~mL}(81 \mathrm{mg} / \mathrm{mL})$. También según FUNASA, todos los lotes liberados pasaron los análisis de control de calidad y ninguno fue rechazado por las dosis de antimonio total, trivalente o pentavalente. 
Riesgos y beneficios del prazicuantel en el tratamiento de la esquistosomiasis en mujeres lactantes y embarazadas

El prazicuantel es el fármaco de elección en el tratamiento de la esquistosomiasis, pero en la actualidad no se recomienda su uso en mujeres en edad fecunda. En abril de 2002, la Organización Mundial de la Salud (OMS) realizó en Ginebra una consulta informal sobre el uso de este fármaco en mujeres lactantes y embarazadas. Las conclusiones se pueden resumir como sigue:

- Las mujeres en edad fecunda no deben ser excluidas de los programas poblacionales de quimioterapia de la esquistosomiasis.

- Las mujeres prepúberes y pospúberes deben ser incluidas en todas las estrategias de control de la esquistosomiasis, y se deben tomar medidas específicas para garantizar su cobertura.

- Se les debe ofrecer tratamiento inmediato a todas las mujeres lactantes y embarazadas con esquistosomiasis.

- Se les debe ofrecer tratamiento a todas las mujeres lactantes y embarazadas que vivan en zonas donde la enfermedad sea altamente endémica y en las que se emplee quimioterapia para toda la población.

- En las zonas endémicas donde no se emplee esta estrategia, las mujeres en edad fecunda (incluidas las embarazadas y las lactantes) deben ser consideradas como un grupo de alto riesgo y se les debe ofrecer tratamiento.

- La OMS debe alentar los programas de control con prazicuantel en embarazadas, con el fin de recoger datos sobre el desenlace materno y fetal en un número suficiente de casos. Estos datos se deben comparar con la incidencia esperada de alteraciones fetales y otros desenlaces adversos del embarazo en esas poblaciones.

\section{Información de seguridad importante para pacientes tratados con celecoxib (Canadá)}

En Canadá, HS/SC ha proporcionado nueva información relacionada con la seguridad del celecoxib, un AINE inhibidor de la COX-2. En un ensayo clínico a gran escala -el Estudio de Seguridad a Largo Plazo del Celecoxib en la Artritis (Celecoxib Longterm Arthritis Safety Study: CLASS)-, se comparó la seguridad gastrointestinal del celecoxib (400 mg dos veces al día), el diclofenaco (75 mg dos veces al día) y el ibuprofeno ( $800 \mathrm{mg}$ tres veces al día) en pacientes con artritis. No se observaron diferencias entre los tres tratamientos con respecto al riesgo de complicaciones ulcerosas (hemorragia gastrointestinal, perforación y obstrucción). El riesgo de complicaciones ulcerosas y úlceras sintomáticas (úlceras con dolor abdominal, dispepsia, náuseas, diarrea o vómitos) fue menor con el celecoxib que con el ibuprofeno, pero no diferente del registrado con el diclofenaco.

\section{El tamoxifeno en la prevención del cáncer de mama y el riesgo de fenómenos tromboembólicos y cáncer endometrial (EE. UU., Reino Unido)}

El tamoxifeno se está utilizando ampliamente en el tratamiento del cáncer de mama. Los resultados preliminares del Estudio Internacional de Intervención en el Cáncer de Mama (International Breast Cancer Intervention Study: IBIS) también aportan datos favorables al uso del tamoxifeno en la prevención del cáncer de mama en mujeres sanas con alto riesgo. Los resultados obtenidos hasta ahora revelan una reducción de un tercio en la incidencia del cáncer de mama, en comparación con el placebo. No obstante, el estudio también indicó que el tamoxifeno puede aumentar el riesgo de tromboembolias, sobre todo durante e inmediatamente después de intervenciones quirúrgicas importantes o durante la inmovilización. En los EE. UU. también se han descrito acontecimientos graves y potencialmente mortales asociados al uso del tamoxifeno con fines preventivos. La incidencia de accidentes vasculares cerebrales ha sido de 1,43 frente a 1,00 por 1000 mujeres-año con el placebo; la de embolias pulmonares, de 0,75 frente a
0,25 ; la de adenocarcinomas endometriales, de 2,20 frente a 0,71, y la de sarcomas uterinos, de 0,17 frente a 0,00.

El Departamento de Salud del Reino Unido ha enviado a todos los directores de salud pública un comunicado urgente en el que señala que: 1) los beneficios del tratamiento del cáncer de mama con tamoxifeno superan claramente sus riesgos; 2) el estudio IBIS proporciona datos favorables a la acción preventiva del tamoxifeno frente al cáncer de mama, aunque su utilización para este fin todavía no ha sido aprobada, a no ser en el contexto de ensayos clínicos, y 3) es necesario realizar un análisis completo de todos los ensayos clínicos para determinar si los beneficios de la acción preventiva superan los riesgos.

En los EE. UU., la FDA y el fabricante han modificado la información para la prescripción de Nolvadex ${ }^{\circledR}$ (tamoxifeno), recomendando a los profesionales sanitarios que comenten los potenciales beneficios y los riesgos de estos acontecimientos adversos graves tanto con sus pacientes con cáncer de mama como con las mujeres con carcinoma ductal in situ que deseen reducir su riesgo de sufrir cáncer de mama invasivo.

\section{ENMIENDAS A LA ROTULACIÓN/CAMBIOS DE FORMULACIÓN}

\section{Revisión de las precauciones a tomar con la acarbosa, el zafirlukast y la vincristina (Japón)}

Las autoridades sanitarias japonesas han ordenado modificar los prospectos de estos tres fármacos para reflejar reacciones adversas graves que se están notificando. La sección de "Precauciones" del prospecto de Glucobay ${ }^{\circledR}$ (acarbosa) incluirá ahora la afirmación de que el fármaco puede causar alteraciones graves de la función hepática, entre ellas hepatitis fulminante; se recomienda la realización de pruebas funcionales hepáticas una vez al mes durante los primeros 6 meses, y a intervalos regulares más largos a partir de entonces. En el caso de Accolate ${ }^{\circledR}$ (zafirlukast) se añadi- 
rán las alteraciones de la función hepática y la ictericia a la lista de reacciones adversas graves, mientras que en el caso de Oncovin ${ }^{\circledR}$ (sulfato de vincristina) se mencionarán la depresión medular y la neumonía intersticial en la sección de "Precauciones".

\section{Cambios importantes en el prospecto de enoxaparina sódica inyectable (EE. UU.)}

La FDA y el fabricante (Aventis Pharmaceuticals) han reforzado la sección de "Advertencias y precauciones" de los prospectos de Lovenox ${ }^{\circledR}$ (enoxaparina sódica inyectable) para informar que no se recomienda su uso en la profilaxis de la trombosis en pacientes con prótesis valvulares cardíacas. La medida se ha tomado después de que se hayan producido varios casos de trombosis de prótesis valvulares cardíacas en pacientes tratados con enoxaparina. Algunos de estos pacientes eran embarazadas en las que la trombosis provocó la muerte de la madre o del feto. Las embarazadas con prótesis valvulares cardíacas pueden correr mayor riesgo de sufrir fenómenos tromboembólicos. A la subsección de "Efectos teratogénicos" se ha añadido un párrafo sobre la notificación de malformaciones congénitas (malformaciones cerebrales, cardíacas y de los miembros, hipospadias, malformaciones vasculares periféricas y displasia fibrótica) en hijos de mujeres que recibieron enoxaparina durante el embarazo. También se ha revisado la subsección de efectos no teratogénicos para señalar que las embarazadas tratadas con anticoagulantes, incluida la enoxaparina, corren mayor riesgo de sufrir hemorragias, que pueden ocurrir en cualquier localización y producir la muerte de la madre o del feto.

\section{Se refuerzan las secciones de "Advertencias" $\mathrm{y}$ "Contraindicaciones" de la ziprasidona (EE. UU.)}

La FDA y el fabricante (Pfizer) han reforzado la sección de "Advertencias y precauciones" del prospecto de Geodon $^{\circledR}$ (ziprasidona) para informar de los fármacos individuales o clases de fármacos cuya asociación está contraindicada. En el nuevo prospecto se afirma claramente que no se puede excluir un efecto aditivo entre la ziprasidona y otros fármacos que prolongan el intervalo QT. Por lo tanto, la ziprasidona no se debe administrar junto con dofetilida, sotalol, quinidina, otros antiarrítmicos de las clases Ia y III, mesoridazina, tioridazina, clorpromazina, droperidol, pimozida, esparfloxacino, gatifloxacino, moxifloxacino, holofantrina, mefloquina, pentamidina, trióxido de arsénico, acetato de levometadilo, mesilato de dolasetrón, probucol ni tacrolimus. También se debe evitar la combinación de ziprasidona con otros fármacos que aumentan el intervalo QTc. Además, los médicos deben estar atentos a la identificación de otros fármacos que aumenten dicho intervalo. Por último, también se debe evitar la administración de ziprasidona en pacientes con síndromes congénitos de alargamiento del intervalo QT o con antecedentes de arritmias cardíacas.

\section{Se enmienda el prospecto de la clozapina para reflejar su asociación a la miocarditis (Canadá)}

En enero de 2002, HC/SC y el fabricante (Novartis Pharmaceuticals) alertaron a los profesionales sanitarios del riesgo de complicaciones cardiovasculares durante el tratamiento con clozapina. La asociación se basó en los datos de farmacovigilancia de cuatro países en los que los pacientes tratados con clozapina son sometidos a vigilancia hematológica. Los datos consistieron en 15 casos de miocarditis (5 de ellos mortales) en 8000 pacientes australianos (incidencia de 96,6/100000 pacientes-año), 7 (1 mortal) en 15000 pacientes canadienses (incidencia de 16,3/100 000), 30 (8 mortales) en 24108 pacientes del Reino Unido (incidencia de $43,2 / 100000)$ y 30 (1 mortal) en 205493 pacientes estadounidenses (incidencia de 5,0/100 000). Posteriormente, Novartis revisó la adver- tencia enmarcada del prospecto de Clozaril $^{\circledR}$ (clozapina) y la cambió de sitio, colocándola al principio de la sección de "Información de prescripción". Esta versión revisada advierte a los profesionales sanitarios de la asociación entre el tratamiento con clozapina y la aparición de casos de miocarditis. En una carta a los profesionales, Novartis los advierte de que deben tener en cuenta la posibilidad de miocarditis en pacientes tratados con clozapina que presenten fatiga inexplicada, disnea, taquipnea, fiebre, dolor torácico, palpitaciones, otros signos o síntomas de insuficiencia cardíaca o signos electrocardiográficos tales como alteraciones de ST-T o arritmias. La taquicardia ha sido identificada como un signo de presentación, por lo que los pacientes con taquicardia durante el primer mes de tratamiento deben ser vigilados cuidadosamente en busca de otros signos de miocarditis. También se les advierte a los médicos que deben interrumpir rápidamente el tratamiento con clozapina ante la sospecha de miocarditis y que no deben volver a administrar el fármaco a los pacientes con miocarditis inducida por clozapina.

\section{DECISIONES DIVERSAS}

\section{La FDA aprueba la comercialización restringida del alosetrón (EE. UU.)}

La FDA aprobó la comercialización del alosetrón en febrero de 2000, pero en noviembre del mismo año, tras la notificación de varios casos graves de colitis isquémica y complicaciones del estreñimiento, algunos de ellos mortales, el fabricante (GlaxoSmithKline) lo retiró voluntariamente del mercado. La FDA ha vuelto a aprobar su comercialización, pero con restricciones. El fabricante pondrá en marcha un programa de gestión del riesgo que, entre otras medidas, creará una lista de médicos interesados en prescribir el fármaco. El programa está destinado a asegurar que los pacientes y los médicos conocen los riesgos y beneficios del alosetrón y que el fármaco solo se prescribirá en casos apropiados. La in- 
dicación se ha restringido al tratamiento de los casos graves de mujeres con síndrome del intestino irritable con predominio de diarrea que no hayan respondido al tratamiento convencional. Al restringir el uso del alosetrón a este grupo de pacientes con afectación grave se procura maximizar la relación entre los riesgos y los beneficios. Los casos de síndrome del intestino irritable considerados graves representan menos del 5\% del total y solo parte de ellos tienen predominio de diarrea.

\section{EVALUACIÓN DE NUEVOS PRODUCTOS}

\section{¿Es necesaria otra estatina (rosuvastatina)? (Canadá)}

Las estatinas comercializadas actualmente en Canadá son seis: atorvastatina, cerivastatina, fluvastatina, lovastatina, pravastatina y simvastatina. Las tres últimas han demostrado reducir la incidencia de infarto de miocardio y muerte por causas cardiovasculares en pacientes con $y$ sin antecedentes de infarto de miocardio. Los efectos adversos notificados con las actuales estatinas suelen ser leves y transitorios. Los más frecuentes consisten en trastornos gastrointestinales, miopatía, aumento de las enzimas hepáticas, fatiga y cefalea. La rabdomiólisis es rara, pero grave, y puede ocurrir con cualquier estatina. Pequeños estudios aleatorizados de comparación entre la rosuvastatina (40 mg) y la atorvastatina $(80 \mathrm{mg})$, la estatina más potente existente actualmente en el mercado, han mostrado tras 6 semanas de tratamiento una reducción similar del colesterol de las lipoproteínas de baja densidad, pero un mayor aumento del colesterol de las lipoproteínas de alta densidad con la rosuvastatina. Los estudios sobre la seguridad de esta última han incluido muy pocos pacientes y han tenido una duración extremadamente corta. En resumen, los estudios realizados hasta la fecha son insuficientes para determinar si la rosuvastatina proporciona alguna ventaja en comparación con otras esta- tinas. Como la seguridad, eficacia y tolerabilidad de los fármacos hipolipidemiantes utilizados en la actualidad en la prevención de la cardiopatía coronaria están bien establecidas, son necesarios más estudios para evaluar con precisión los beneficios y los riesgos relativos de la rosuvastatina.

\section{Otro análogo de las prostaglandinas (travoprost) para el tratamiento del glaucoma (Australia)}

En Australia se ha aprobado el uso de la solución de travoprost al 0,004\% (envases de 2,5 mL) para el tratamiento de la hipertensión intraocular. El travoprost se añade así a los análogos de las prostaglandinas ya aprobados para esta indicación, como el latanoprost, que se está usando ampliamente en estos casos. Como el travoprost es un análogo de la prostaglandina $\mathrm{F}_{2 \alpha^{\prime}}$ reduce la presión intraocular aumentando el flujo del humor acuoso. Solo se necesita una aplicación diaria, puesto que el efecto dura al menos 24 horas. En un ensayo clínico se compararon 801 pacientes tratados durante un año con travoprost, latanoprost o timolol. La presión intraocular disminuyó en un $30 \%$ o por debajo de $17 \mathrm{~mm}$ $\mathrm{Hg}$ en el $54,7 \%$ de los pacientes tratados con travoprost, en el $50 \%$ de los tratados con latanoprost y en el 39\% de los tratados con timolol. La presión intraocular media con el travoprost fue $0,8 \mathrm{~mm} \mathrm{Hg}$ menor que con el latanoprost. Otro estudio confirmó que el travoprost tiene un efecto significativamente mayor que el timolol. Más del $37 \%$ de los pacientes tratados con travoprost pueden sufrir hiperemia ocular durante el tratamiento, frecuencia que es superior a la observada con el latanoprost o el timolol. Otros efectos adversos oculares son el picor y las alteraciones de las pestañas. Además, el travoprost puede producir alteraciones del color del iris, a veces permanentes. El travoprost se puede utilizar como monoterapia o en combinación con el timolol. Cuando el paciente esté tomando ambos fármacos, las instilaciones de uno y otro deben realizarse con un intervalo de al menos 5 minutos.
Aunque la eficacia del travoprost es similar a la del latanoprost, los efectos adversos locales pueden reducir su aceptación por parte de los pacientes.

\section{POLÍTICAS DE MEDICAMENTOS}

\section{Negociaciones entre los países \\ del Caribe y la industria farmacéutica para bajar los precios de los antirretrovíricos en la región}

En el marco de la Iniciativa de Acceso Acelerado, 16 países caribeños (los de la comunidad del Caribe - CARICOM - y la República Dominicana), han completado con éxito una serie de negociaciones de precios con los representantes de los fabricantes de antirretrovíricos para el tratamiento de la infección por el virus de la inmunodeficiencia humana (VIH). Las negociaciones culminaron durante la XIV Conferencia Internacional sobre el $\mathrm{VIH} /$ sida, que tuvo lugar en Barcelona en julio de 2002, con la firma de una declaración de intenciones conjunta por parte de la CARICOMRepública Dominicana y las compañías farmacéuticas. Con anterioridad se habían realizado en el Caribe y en la sede de la OMS, en Ginebra, una serie de reuniones técnicas y de negociación de precios facilitadas por la Organización Panamericana de la Salud/OMS y ONUSIDA. Durante la presentación del documento marco a las compañías farmacéuticas, se buscó que estas se comprometieran a considerar al Caribe como una región, y no como un grupo de países individuales. En discusiones técnicas posteriores, la industria farmacéutica expresó su apoyo a la iniciativa regional y al fortalecimiento de las alianzas publicoprivadas en la región. Durante las negociaciones de precios que se siguieron, los países del Caribe alcanzaron un éxito histórico al conseguir por primera vez un precio regional uniforme bajo (1 152 dólares estadounidenses: USD) para el tratamiento de primera línea (zidovudina + lamivudina + nevirapina). Este precio es similar al de los países del África Subsahariana y es válido para toda la región (CARICOM y República Domi- 
nicana), independientemente de los índices de desarrollo de los países miembros, y representa una reducción de aproximadamente un $90 \%$ en comparación con el costo del mismo tratamiento en los EE. UU. (USD 10 500). Además, también se ofreció una segunda opción para el tratamiento de primera línea (zidovudina + lamivudina + efavirenz) con una reducción de costo también del $90 \%$, aunque en este caso se decidió que este precio reducido (USD 1220 ) no será aplicable en Barbados ni en las Bahamas.

\section{Nuevos países participantes en el Programa Internacional de Farmacovigilancia de la OMS}

El número de países participantes en el Programa Internacional de Farmacovigilancia de la OMS sigue aumentando. Los últimos en unirse a él han sido Letonia y Perú, con lo cual se eleva a 67 el número de miembros oficiales. También se han recibido solicitudes de adhesión por parte de Bahrein y Moldavia, países que, mientras no reciban su estatus de miembro formal, serán considerados como miembros asociados, esto es, países con fuerte capacidad de farmacovigilancia, pero sin un centro de vigilancia de reacciones adversas a los fármacos reconocido formalmente.

\section{REFERENCIAS}

WHO Information Exchange System. Alert No. 105. June 2002.

FDA/MEDWATCH, 2002. Safety Information Summaries. [Sitio en Internet] FDA. Disponible en: http://www.fda.gov/medwatch/ SAFETY/2002/safety02.htm\#drugs. Acceso el 20 agosto 2002.

FDA Talk Paper, June 2002. [Sitio en Internet] FDA. Disponible en: http://www.fda.gov/ opacom/hpnews.html. Acceso el 20 agosto 2002.

WHO Pharmaceuticals Newsletter 2002;2:112. Disponible en: http://www.who.int/ medicines/library/pnewslet/2news2002.pdf. Acceso el 20 agosto 2002.

ANVISA. Alerta Ufarm/Anvisa No. 11, 05/06/02. [Sitio en internet] ANVISA. Disponible en: http://www.anvisa.gov.br/farmacovigilancia/index.htm. Acceso el 20 agosto 2002.

Committee on Safety of Medicines/MCA. Current Problems in Pharmacovigilance 2002:3.

MEDSAFE Prescriber Update 2002;23:20.

MEDSAFE Prescriber Update 2002;23:21.

MEDSAFE Prescriber Update 2002;23:24.

Health Canada/Santé Canada Online, 2002. Warnings/Advisories. [Sitio en Internet] Health Canada/Santé Canada. Disponible en: http:// www.hc-sc.gc.ca/english/protection/warnings/ 2002.htm. Acceso el 20 agosto 2002.

Uppsala Monitoring Centre Homepage. What's new?, 2002. [Sitio en Internet] WHO-UMC. Disponible en: http://www.who-umc.org/ umc.html. Acceso el 20 agosto 2002.
Australian Prescriber. 2002;25(2):29-31. Australian Prescriber. 2002;25(2):47. Australian Prescriber. 2002;25(3):75.
Información farmacológica da a conocer las decisiones oficiales sobre regulación de productos farmacéuticos adoptadas por organismos gubernamentales e internacionales en todo el mundo, los fundamentos científicos en que se sustentan tales decisiones y otros datos de interés relacionados con el tema. Como la mayor parte de la información proviene de fuentes de circulación relativamente limitada, su diseminación en esta forma permite hacerla llegar a un público más amplio. De esta manera se pretende contribuir a limitar el uso irracional de medicamentos y fomentar su uso racional en la Región de las Américas. La sección está a cargo del Programa de Medicamentos Esenciales y Tecnología para la Salud (HSE) de la OPS y se publica en la Revista Panamericana de Salud Públical Pan American Journal of Public Health con regularidad. Las separatas pueden solicitarse al programa mencionado, Organización Panamericana de la Salud, 525 Twenty-third Street, NW, Washington, DC 20037, EUA.

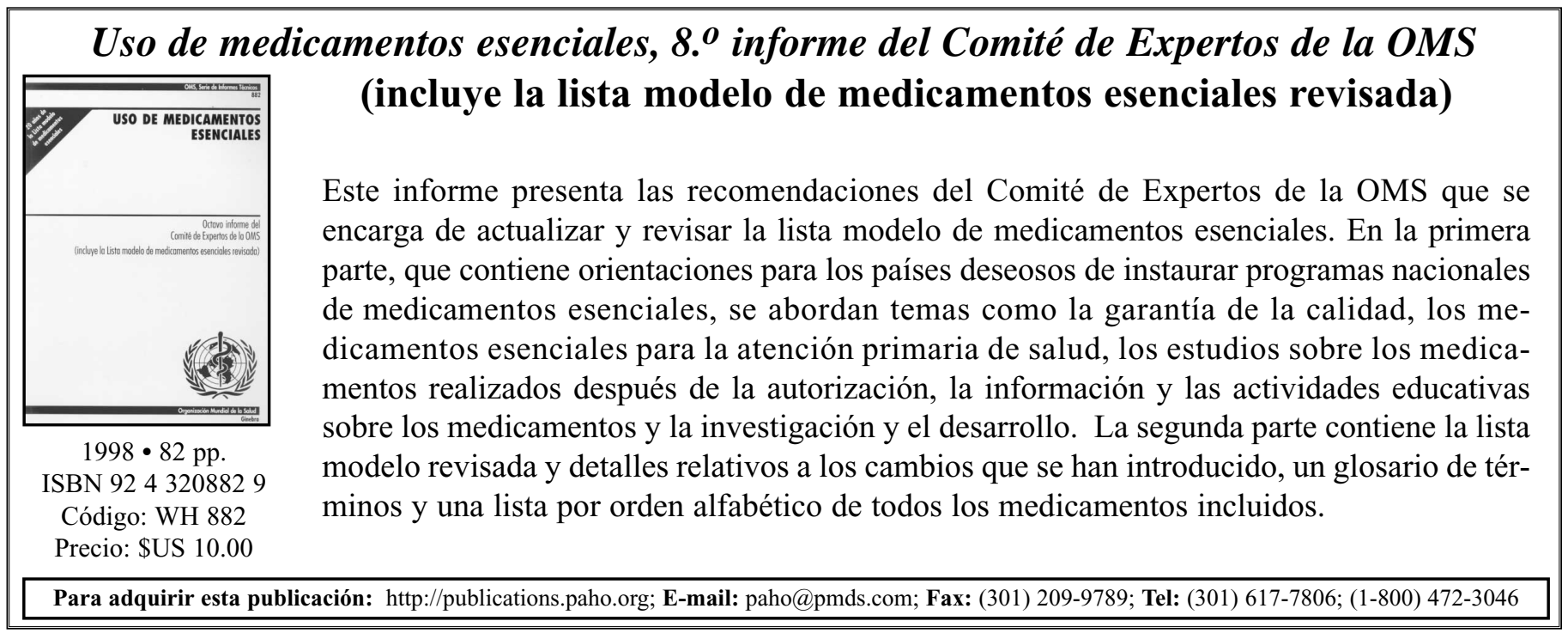

